\section{Estimação da mortalidade infantil no contexto de descentralização do Sistema Único de Saúde (SUS)}

\section{Estimating infant mortality in a decentralized National Health System}

Paulo Germano de Frias 1

Célia Landman Szwarcwald 2

Pedro Israel Cabral de Lira 3

\begin{abstract}
The article discusses strategies for estimating infant mortality in the decentralized Brazilian National Health System. It presents direct and indirect techniques for measuring infant mortality, the ways these can be applied and the methodological problems that arise. It further discusses the Ministry of Health's permanent life events information systems and the strategies for evaluating the adequacy of information for the calculation of infant mortality. These issues are set in the context of the new needs that have emerged from the decentralization of the Brazilian health system. Finally, it remarks on the challenge of direct estimation of infant mortality using Ministry of Health information systems in small-scale municipalities and the shortcomings of the data available.
\end{abstract}

Key words Infant mortality, Information systems, Health evaluation, Decentralization, Estimation techniques, Vital statistics, Brazil

\author{
${ }^{1}$ Instituto de Medicina Integral Professor Fernando Figueira. Rua dos \\ Coelhos, 300. Boa Vista. Recife, PE, Brasil. CEP: 50.070-590. \\ E-mail: pfrias@imip.org.br \\ ${ }^{2}$ Laboratório de Informações em Saúde. Instituto de Comunicação e \\ Informação Científica e Tecnológica em Saúde. Fundação Oswaldo \\ Cruz. Rio de Janeiro, RJ, Brasil. \\ 3 Departamento de Nutrição. Universidade Federal de Pernambuco. \\ Recife, PE, Brasil.
}

\section{Resumo}

$O$ artigo aborda as estratégias para estimação da mortalidade infantil no contexto da descentralização do sistema de saúde brasileiro. Apresenta as técnicas de mensuração diretas e indiretas da mortalidade infantil, as possibilidades de aplicação e os problemas metodológicos. Discute os sistemas de informação sobre eventos vitais de registro contínuo do Ministério da Saúde e estratégias para a avaliação da adequação das informações para a estimação da mortalidade infantil. Estes aspectos são contextualizados à luz das novas necessidades que emergiram com o processo de descentralização do sistema de saúde brasileiro. Nas considerações finais, são apontados os desafios para a estimação da mortalidade Infantil pelo método direto a partir dos sistemas de informações do MS em municípios de pequeno porte e precariedade das informações vitais.

Palavras-chave Mortalidade infantil, Sistemas de informação, Avaliação em saúde, Descentralização, Técnicas de estimação, Estatísticas vitais, Brasil 


\section{Introdução}

No contexto de reformas do setor saúde brasileiro, a influência que a conjuntura nacional exerce na definição das políticas de saúde e na determinação de modelos assistenciais é plenamente reconhecida. Com a Constituição de 1988, houve a inovação quanto à autonomia aos três entes federados, competências e descentralização política e administrativa. Os municípios adquiriram status valorizado e, objetivando a integração e a interdependência entre os três poderes, foram definidas responsabilidades e partilha de recursos para a realização de ações governamentais descentralizadas. ${ }^{1,2}$

É hoje um grande desafio assegurar o cumprimento do princípio constitucional, com o envolvimento da União, das 27 Unidades da Federação (UF) e dos mais de cinco mil municípios distribuídos em território de dimensões continentais, com desigualdades regionais profundas e sem história de relações intergovernamentais cooperativas. ${ }^{1}$ A necessidade de ordenamento do processo de implantação do Sistema Único de Saúde (SUS), com a definição de sua base legal, surge, neste cenário, como uma das maiores políticas públicas universais. ${ }^{2}$

Com a instituição do SUS brasileiro afloraram necessidades de saúde, relacionadas às mudanças demográficas e epidemiológicas, e organizativas para suprir deficiências históricas. 1,2 $\mathrm{O}$ avanço do processo de implantação do sistema, caracterizado pela descentralização das ações de saúde, e o estabelecimento de pactos entre os entes federados favoreceram o aumento da demanda por informações que possibilitassem o conhecimento do perfil epidemiológico, o planejamento e a avaliação das ações desenvolvidas. ${ }^{3}$ A partir deste marco, os sistemas de informação do Ministério da Saúde (MS) incorporaram usuários e passaram a ter maior aplicação na gestão do sistema de saúde.3,4

O Sistema de Informações sobre Mortalidade (SIM) e o Sistema de Informações sobre Nascimentos (Sinasc), sistemas de racionalidade epidemiológica, ganharam relevância por disporem de dados essenciais para o cálculo de indicadores de monitoramento da situação de saúde e de avaliação de ações programáticas. ${ }^{5}$ Nesse contexto, a mortalidade infantil, definida, conceitualmente, como o número de mortes para cada mil nascidos vivos, ganha especial destaque por expressar as condições de vida e de saúde, o acesso aos serviços de saúde, e o desempenho dos programas dirigidos à sua redução. Referência na área de saúde pública, a diminuição da mortalidade infantil é alvo de diversas pactuações nacionais e internacionais. 5
Nas últimas décadas, vários programas têm sido desenvolvidos pelo Ministério da Saúde com forte potencial de atuação na redução da mortalidade infantil. Entre eles, destacam-se os programas:6,7 Programa de Imunização (1975); o Programa de Incentivo ao Aleitamento Materno (1981); o Programa de Assistência Integral à Saúde da Mulher e da Criança (1984); o Programa de Redução da Mortalidade Infantil (1995); e, como parte integrante da política nacional de atenção básica, o Programa de Agentes Comunitários de Saúde (1991) e o Programa de Saúde da Família (1994), com os objetivos de aumentar a acessibilidade ao sistema de saúde e incrementar as ações de prevenção e promoção da saúde. Mais recentemente, foram implementados o Pacto Nacional pela Redução da Mortalidade Materna e Neonatal e o projeto Rede Cegonha.6,7

Desde então, os municípios desenvolveram propostas buscando viabilizar e otimizar a implantação desses programas, com critérios de organização definidos pelo governo federal e outros próprios de gestão municipal e estadual, levando a necessidade de avaliar os processos de desenvolvimento e execução das intervenções e os resultados sobre a saúde da população. ${ }^{6}$ Como expressão da efetividade das ações, 3,5 o monitoramento da mortalidade infantil torna-se fundamental, e traz à tona o problema de estimação deste indicador nos municípios brasileiros.

Diante das restrições no uso de técnicas demográficas de mensuração indireta para a estimação da mortalidade infantil por município, conforme apontadas anteriormente, ${ }^{8}$ as estimativas pelo método direto ganharam importância e renovaram o interesse pelas informações vitais de registro contínuo. 9,10 Não somente foram propostos métodos de avaliação dos dados dos sistemas de informações vitais do MS8,11-13 como também o MS adotou uma série de iniciativas para a melhoria da cobertura e da qualidade das informações vitais. 14 Entretanto, o acompanhamento das tendências temporais deste indicador em áreas com baixo contingente populacional e com precariedade de informações vitais permanece ainda como um desafio a ser superado. 15

O presente trabalho tem como objetivo discutir as estratégias utilizadas para estimar a mortalidade infantil, no contexto de descentralização do SUS e a consolidação do processo de municipalização. 
Estratégias para a estimação da mortalidade infantil no Brasil

Os indicadores epidemiológicos dos problemas relacionados à saúde, a exemplo da mortalidade infantil, são instrumentos indispensáveis para definir as prioridades nas políticas públicas, planejar ações e serviços, monitorar a situação de saúde e avaliar o desempenho do sistema de saúde. Entretanto, problemas no cálculo dos indicadores podem gerar interpretações equivocadas, restringindo o uso da informação para o aperfeiçoamento das políticas e programas de saúde.16 Questões relacionadas à cobertura, regularidade, natureza e processo de coleta dos dados, técnicas de aferição e de estimação são algumas das dificuldades. $4,15,17$

No que se refere à mortalidade infantil, o cálculo do indicador pelo método direto, a partir de dados dos sistemas de informações vitais do MS, não tem sido recomendado em Estados onde há precariedade dos registros de óbitos e nascimentos. 13 Por isso, em alguns Estados do Brasil, persiste a estimação indireta da mortalidade infantil, com base em método proposto pelo Instituto Brasileiro de Geografia e Estatística (IBGE).18 A seguir, descrevem-se os métodos usados para a estimação da mortalidade infantil, no Brasil.

\section{Técnicas demográficas de estimação}

indireta

Devido às limitações nas fontes primárias de informação, a partir de meados do século XX, a pesquisa demográfica dedicou-se à formulação de técnicas de estimação indireta de indicadores de mortalidade, de forma a substituir as estimativas diretas, passíveis de graves erros em situações de coberturas incompletas das estatísticas vitais. ${ }^{19}$

Originalmente proposto por Brass, ${ }^{20}$ o método é baseado em relacionar proporções de filhos sobreviventes, classificadas por grupo quinquenal de idade das mães, às probabilidades de morte do nascimento a diferentes idades na infância, a partir da inclusão de perguntas sobre o número total de filhos nascidos vivos e o número de filhos vivos atualmente (ou sobreviventes) nos censos ou pesquisas por amostragem. ${ }^{21}$ A partir da década de 1970, em grande parte dos países da América Latina, tornou-se habitual incluir perguntas de caráter retrospectivo nos censos e pesquisas domiciliares, destinadas especificamente à mensuração indireta da mortalidade. 22

O procedimento original de Brass 20 foi aperfeiçoado ao longo dos anos, no que se refere, principalmente, aos modelos de mortalidade e de fecundi- dade utilizados para a conversão das proporções de filhos sobreviventes em probabilidades de morte. Adaptações posteriores incluíram situações de mortalidade variável com o tempo e relacionaram probabilidades de sobrevivência dos filhos, categorizadas por grupos de idade da mãe, a tendências temporais da mortalidade infantil em anos anteriores à pesquisa, solucionando o problema de corresponder um ponto do tempo à estimativa da mortalidade infantil.23-25

Atualmente, as estimativas da mortalidade infantil fornecidas pelo IBGE, são realizadas pelo método demográfico indireto, a partir das informações coletadas nos censos e nas Pesquisas Nacionais de Amostra por Domicílio (PNAD). Este método tem sido utilizado em publicações acadêmicas, 15 como também por organismos oficiais para substituir a técnica de mensuração direta. 26

Em países com ausência ou precariedade de registro das informações vitais, a estimação da mortalidade infantil é baseada em informações coletadas em inquéritos domiciliares sobre a história completa de nascimentos entre mulheres em idade fértil. A estimação é feita por meio de uma tábua de vida, onde as probabilidades de morte são estimadas pelas informações sobre todos os nascimentos e mortes relatadas pelas mães e o número de crianças de um determinado grupo etário exposto ao risco de morrer em um certo período de tempo. 27

A Pesquisa de Demografia e Saúde (Demographic and Health Survey - DHS), realizada periodicamente em vários países do mundo, utiliza uma variante deste método, conhecido como "synthetic cohort life table approach". O procedimento é, entretanto, sujeito ao viés de memória e resulta em uma subestimação progressiva da mortalidade com o aumento do número de anos que precedem o inquérito, já que erros no relato das datas de nascimento e morte ocorrem com mais frequência com o passar do tempo. 28

No Brasil, a Pesquisa Nacional de Demografia e Saúde (PNDS) da criança e da mulher foi realizada em 1986, 1996, e 2006.29 Porém, apesar das estimativas publicadas, o uso dos dados da PNDS para a estimação da mortalidade infantil é pouco frequente e não é adotado oficialmente, provavelmente pelo pequeno tamanho de amostra, o que implica na impossibilidade de qualquer desagregação geográfica das estimativas. 29

Vários problemas têm sido apontados na estimação da mortalidade infantil por meio de métodos baseados em pesquisas domiciliares. A qualidade das estimativas depende fortemente do tamanho de amostra, do desenho do estudo e da forma como é 
conduzido o inquérito. Além disso, apesar de serem provenientes de amostras da população, as estimativas são tratadas como parâmetros populacionais, sem estimação do erro das estimativas médias e sem consideração do efeito de desenho. ${ }^{12,30}$

No que diz respeito à estimação da mortalidade infantil no nível municipal, as estimativas elaboradas a partir de pesquisas por amostragem, devido às limitações no tamanho da amostra, não podem ser fornecidas em pequenas áreas, o que restringe o monitoramento da situação de saúde infantil em municípios de baixo contingente populacional. Adicionalmente, não tendo o caráter de continuidade, peculiar aos dados de registro, este tipo de mensuração da mortalidade infantil fornece estimativas que se referem a um período de dois a três anos antes da pesquisa, dificultando a avaliação imediata da efetividade de ações e programas de saúde dirigidos, especificamente, às crianças menores de um ano de idade. 31

Em 1999, por solicitação do MS, as taxas de mortalidade infantil foram estimadas por técnicas de mensuração indireta em todos os municípios brasileiros com mais de 80.000 habitantes. ${ }^{26}$ As taxas foram apresentadas para todos os estados e capitais, incluindo aquelas com dados adequados para o cálculo direto. Nessas áreas, em decorrência do procedimento metodológico empregado, as taxas apresentadas superestimavam os valores das taxas de mortalidade infantil. As estimativas tiveram, pois, impacto negativo e de desestímulo entre os gestores de saúde locais, que vinham desenvolvendo ações especificamente dirigidas à diminuição dos óbitos infantis. 12

Outras limitações das técnicas de estimação indireta realizadas por meio de pesquisas domiciliares referem-se à omissão de perguntas diretas relevantes e/ou inadequado preenchimento do questionário, a exemplo da idade da mãe, dado fundamental na aplicação do método. 18 No censo demográfico de 2001, omitiu-se a data de óbito das crianças nascidas nos 12 meses anteriores à pesquisa. A quebra dos pressupostos do método originalmente proposto por Brass, ${ }^{20}$ como mudanças acentuadas na fecundidade e nos movimentos migratórios, pode também interferir nos resultados da estimação. ${ }^{15,18}$

Um problema adicional, que ocorre com frequência na estimação indireta da mortalidade infantil, é a variabilidade das estimativas, seja pelo modelo de tábuas de vida adotado 32 ou pelo método utilizado. 33 Igualmente, dependendo das perguntas utilizadas nos inquéritos nacionais, as estimativas podem ser díspares, ${ }^{9}$ dificultando a interpretação das tendências temporais e o conhecimento do nível real da mortalidade infantil na população sob estudo. 34

Para minimizar as limitações do método indireto de estimação, para as estimativas da Rede Interagencial de Informações para a Saúde (Ripsa), é utilizado um modelo denominado de "mix". ${ }^{13}$ Neste modelo, para as Unidades da Federação cujas informações de nascidos vivos e óbitos são consideradas adequadas, a taxa de mortalidade infantil é calculada pelo método direto, enquanto naquelas UF com informações insatisfatórias, é usado o método indireto proposto pelo IBGE. 13,35 Comparando as estimativas para o ano de 2007, a mortalidade infantil diminui de 24,3 para 20,0 por $1000 \mathrm{MV}$, utilizandose, respectivamente, técnicas indiretas de estimação para todos os Estados e o modelo "mix" adotado pela Ripsa.

\section{Cálculo direto da mortalidade infantil}

Por definição, o coeficiente de mortalidade infantil (CMI) é calculado pelo número de mortes no primeiro ano de vida para cada 1000 nascidos vivos. O MS dispõe de dois sistemas de informações para o cálculo do CMI: o Sistema de Informações sobre Mortalidade (SIM), que foi implantado em 1975, e o Sistema de Informações sobre Nascimentos (Sinasc), a partir de 1990.17

O SIM, o sistema de informações mais antigo do MS, capta informações sobre as características sociais, demográficas e epidemiológicas dos óbitos, 36 possibilitando o monitoramento, e um maior detalhamento da mortalidade e seus determinantes para diversos níveis de agregação geográfica.

A Declaração de Óbito (DO) é o instrumento oficial de coleta de dados do SIM e deve ser preenchida pelo médico. O fluxo de encaminhamento dessas declarações, bem como as normas quanto ao seu preenchimento e o processamento das informações são definidos pelo MS, e estão detalhados nos manuais de procedimentos e de preenchimento da DO. O MS detém a gestão nacional do sistema e é responsável pela consolidação e divulgação dos dados. ${ }^{36}$ Estes são disponibilizados por município brasileiro, desde 1979, por meio de consulta na internet através do site do DATASUS (www.datasus.gov.br).

O Sinasc, concebido a partir da experiência do SIM, teve sua implantação gradual entre as Unidades da Federação. Os dados para o Brasil são disponíveis a partir de 1994.17 O Sinasc disponibiliza não só informações sobre nascimentos, mas também sobre as mães, tais como características do parto, da gravidez, e do recém-nascido, permitindo obter um panorama epidemiológico e sócio-espacial das condições de saúde materno-infantil. 4 
A Declaração de Nascido Vivo (DN) é o instrumento oficial de coleta de dados do Sinasc. O fluxo de encaminhamento das DN, bem como as normas quanto ao seu preenchimento e o processamento das informações são definidos pelo MS, gestor nacional do sistema, e estão descritos nos manuais de procedimento e preenchimento da DN.4,17 O processo de divulgação dos dados é similar ao adotado para o SIM e está disponível no site do DATASUS (www.datasus.gov.br). ${ }^{4}$

Desde a implantação dos sistemas, o SIM e Sinasc vêm sendo aperfeiçoados e atualizados com frequência, através de normas e regulamentações publicadas pelo MS, pactuadas com as representações das esferas de governo integrantes do SUS. 17,37

$\mathrm{Se}$ as estatísticas vitais no Brasil fossem completas em todas as UF, o método direto seria, sem dúvida, o preferencial para estimar a mortalidade infantil. ${ }^{13}$ Entretanto, devido à sub-enumeração das mortes e de nascidos vivos em algumas áreas do país, o CMI não pode ser calculado pelo método direto em todo o território nacional. De acordo com os critérios estabelecidos pela Ripsa, em apenas oito UF - Espírito Santo, Rio de Janeiro, São Paulo, Paraná, Santa Catarina, Rio Grande do Sul, Mato Grosso do Sul e Distrito Federal - pode-se calcular a mortalidade infantil pelo método direto. 35

A cobertura incompleta e a falta de regularidade da informação em algumas áreas são os maiores empecilhos para o uso direto dos dados no cálculo do CMI.9,17 De acordo com estimativas indiretas do IBGE, os dois sistemas ainda apresentam coberturas incompletas, sendo apenas de $90 \%$ para os óbitos totais e $71 \%$ para os óbitos de menores de um ano do SIM e de $92 \%$ para o Sinasc, no Brasil como um todo em 2008.35 Porém, apresentando o mesmo padrão de desigualdades socioeconômicas vigente no país, as menores coberturas ocorrem nas regiões Nordeste, Norte e alguns Estados do Centro-Oeste. 35

Limitações adicionais são relacionadas a erros nos registros, destacando-se os relativos à definição dos eventos, como nascido vivo, nascido morto, perda fetal e aborto. A falta de rigor no registro do evento pode levar a alterações expressivas de alguns indicadores. 10,17,38 Incorreções do local de ocorrência e residência no registro da informação acarretam, igualmente, a sub ou a superestimação do indicador.

Outro problema reside na estimação do CMI em municípios pequenos. 26 Por ser um evento raro, muitas vezes a ausência de óbitos infantis em um determinado ano não significa CMI igual a zero, mas, simplesmente, que não houve chance do evento ocorrer. Flutuações nas estimativas são frequentemente encontradas, sem refletir, entretanto, irregularidades no registro das informações.

Avaliação das informações sobre nascidos vivos e óbitos dos sistemas do Ministério da Saúde

O MS vem empreendendo esforços para a melhoria dos seus sistemas de informações vitais. $14 \mathrm{O}$ reconhecimento da importância de monitoramento das informações sobre óbitos e nascimentos no nível municipal, aliado à responsabilidade partilhada das três esferas de gestão bem como à facilidade de acesso aos dados, têm resultado no aumento substancial na cobertura e na qualidade das informações de ambos os sistemas. ${ }^{3,17}$ Além disso, a interpretação da estimativa do CMI pelo método direto e sua aplicabilidade no monitoramento e avaliação da situação de saúde têm conferido maior utilidade aos dois sistemas e conseqüente interesse em se apropriar dos seus princípios, diretrizes e modo de operar. 9,10

Com a renovação do interesse das informações vitais, foram propostos métodos na literatura nacional recente para avaliação dos sistemas de informações disponíveis. Lima et al., 39 revisando estudos brasileiros que abordaram dimensões de avaliação da qualidade dos dados, mostraram que o SIM e o Sinasc detiveram o maior número de avaliações. Entre os métodos aplicados, está o relacionamento entre sistemas de informação do MS, a comparação com outros sistemas, a consistência de indicadores do próprio banco de dados, a investigação de irregularidades temporais, e a busca ativa de eventos. $8,11,14,17$

A Ripsa, concebida para aperfeiçoar a capacidade nacional de produção e uso de informações para políticas de saúde, divulga uma matriz de indicadores básicos incluindo o CMI. ${ }^{13}$ Para contornar o problema de inadequação das informações vitais em alguns estados, foi criado um índice de consistência dos dados de registro administrativo. 40 Este índice composto $^{13}$ (p.109), que analisa a cobertura e regularidade do SIM e Sinasc, é o parâmetro utilizado para avaliação dos dados e para estabelecer o método de estimação do CMI a ser utilizado (direto ou indireto) em cada UF.40

\section{Desafios e perspectivas}

Os desafios para a análise da situação das condições de saúde entre as crianças menores de um ano no contexto da descentralização do sistema de saúde brasileiro relacionam-se, certamente, aos métodos de estimação da mortalidade infantil no nível de 
município.

As limitações das técnicas de estimação indireta são relacionadas, 8,18 sobretudo, à impossibilidade de avaliar a efetividade de intervenções implementadas em curto prazo e em distintos níveis de desagregação geográfica, e remetem à necessidade de reflexões adicionais quanto a sua utilidade para a tomada de decisão em saúde coletiva no cenário de descentralização do sistema de saúde. ${ }^{3}$ Por outro lado, investigações relacionadas à avaliação da adequação das informações para estimar a mortalidade infantil,11-13 mostram ainda deficiências importantes nos sistemas de informações vitais. A omissão do registro do óbito compromete o dimensionamento da realidade e a identificação dos fatores que influenciam a mortalidade infantil no nível local.

A estratégia de busca ativa de óbitos e nascimentos tem sido usada para identificar eventos não informados ao sistema de saúde, como também para estabelecer problemas existentes na operacionalização dos sistemas de informações SIM e Sinasc que acarretam o sub-registro das informações vitais. 8,14 Contudo, as limitações do processo de busca ativa se acentuam quando se trata da investigação de óbitos infantis, sobretudo nos municípios de pequeno porte populacional. Por ser um evento raro, a busca precisa ser muito mais intensa para se encontrar poucos eventos. As flutuações nas estimativas são também muito maiores e podem variar, substancialmente, com o achado de um ou dois óbitos infantis.

Tais restrições, entretanto, não se referem apenas às limitações do processo de busca ativa, mas também ao cálculo da mortalidade infantil em pequenas áreas. Questões metodológicas permanecem carecendo de aprofundamento em cenários onde há precariedade de registros dos eventos vitais, $9,14 \mathrm{e}$ em unidades geográficas menores, como os municípios e microrregiões com número pequeno de habitantes. ${ }^{26}$

Para os três entes federados permanece, pois, o desafio de intensificar a ampliação da cobertura, regularidade e qualidade dos dados dos sistemas de informações vitais em tempo oportuno. Além disso, incorporar o uso da informação como elemento indispensável na tomada de decisão no cotidiano da gestão, favorecendo a institucionalização do monitoramento e avaliação do sistema de saúde no nível

\section{Referências}

1. Viana ALA, Machado CV. Descentralização e coordenação federativa: a experiência brasileira na saúde. Ciênc Saúde Coletiva. 2009; 14: 807-17. local é, igualmente, fundamental.

Em anos recentes, o MS tem investido na melhoria dos sistemas de informações vitais, destacando-se: a regulamentação das atividades desenvolvidas pelas três esferas de governo; a divulgação dos dados por município em CD-ROM e na internet; a elaboração de publicações técnicas; a capacitação de codificadores de causa básica; a contratação de consultores nos estados com vistas ao resgate de informações e redução dos óbitos sem definição da causa básica; e a inclusão de metas relacionadas ao SIM e ao Sinasc à Programação Pactuada e Integrada. Outros projetos específicos foram também desenvolvidos, como o estudo de busca ativa de óbitos infantis em municípios das regiões Norte e Nordeste 14 e a elaboração do Sistema de Monitoramento de Indicadores Relacionados à Mortalidade Infantil (MONITORIMI).

Mais recentemente, no ano de 2010, em iniciativa conjunta da Secretaria de Vigilância Sanitária/Ministério da Saúde e Fundação Oswaldo Cruz/Ministério da Saúde, foi conduzida uma pesquisa de busca ativa de óbitos e nascimentos em 133 municípios da Amazônia Legal e do Nordeste, com o objetivo principal de estimar as coberturas do SIM e do Sinasc por estrato amostral, constituído pela categoria de porte populacional do município, região e nível de adequação das informações vitais. Os resultados fornecerão os fatores de correção das informações vitais por município brasileiro.

Progressos foram alcançados, sem dúvida, no Brasil, tanto no que se refere à ampliação da cobertura e da qualidade das informações como também à disponibilidade de métodos de correção das estatísticas vitais, a partir de procedimentos de avaliação da adequação das informações do SIM e do Sinasc. Embora bem melhores do que as técnicas de estimação indireta, tanto do ponto de vista técnico como do político, não são ainda a solução desejada, uma vez que estão sujeitos a erros de estimativa e limitações dos procedimentos utilizados.

Somente a partir da institucionalização da avaliação da situação de saúde local e do uso de procedimento de captação dos eventos vitais pelo próprio município, com ênfase nos locais de ocorrência, ocorrerá o fortalecimento dos sistemas de informação, permitindo o cálculo direto da mortalidade infantil em todo o território nacional.

2. Paim J, Travassos C, Almeida C, Bahia L, Macinko J. The Brazilian health system:history, advances, and challenges. Lancet. 2011; 377: 1778-97. 
3. Branco MAF. Informação em saúde como elemento estratégico para a gestão. In: Gestão Municipal de Saúde: textos básicos. Brasília: Ministério da Saúde; 2001. p. 163 9.

4. Almeida MF, Alencar GP, Schoeps D. Sistema de Informação sobre Nascidos Vivos- Sinasc: uma avaliação de sua trajetória. In: A experiência brasileira em sistemas de informação em saúde. Produção e disseminação sobre saúde no Brasil. Brasília: Ministério da Saúde; 2009. v. 1. p. 11-37.

5. Barros FC, Matijasevich A, Requejo JH, Giugliani E, Maranhão AG, Monteiro CA. Recent trends in maternal, newborn, and child health in Brazil: progress toward millennium development goals 4 and 5. Am J Public Health. 2010; 100: 1877-89.

6. Frias PG, Mullachery PH, Giugliani ERJ. Políticas de Saúde direcionadas às crianças brasileiras: breve histórico com enfoque na oferta de serviços de saúde. In: Saúde Brasil 2008. 20 anos de Sistema Único de Saúde (SUS) no Brasil. Brasília: Ministério da Saúde. 2009; p. 85-110.

7. Victora CG, Aquino EML Leal MC, Monteiro CA, Barros FC, Szwarcwald CL. Maternal and child health in Brazil: progress and challenges. Lancet. 2011; 377: 1863-76.

8. Szwarcwald CL, Andrade CLT, Souza Junior PRB. Estimação da mortalidade infantil no Brasil: o que dizem as informações sobre óbitos e nascimentos do Ministério da Saúde? Cad Saúde Pública. 2002; 18: 1725-36

9. Romero DE. Avaliação dos critérios para o cálculo direto da taxa de mortalidade infantil. Textos para discussão, n. 5 In: Secretaria de Estado de Planejamento e Gestão/ Programa Estado para Resultados; 2009.

10. Becker R. O que há por trás dos dados? In: Salas de situação em saúde: compartilhando as experiências do Brasil. Brasília: Ministério da Saúde / Organização Pan-Americana da Saúde; 2010. p. 39-43

11. Andrade CLT, Szwarcwald CL. Desigualdades sócio-espaciais da adequação das informações de nascimentos e óbitos do Ministério da Saúde, Brasil, 2000-2002. Cad Saúde Pública. 2007; 23: 1207-16.

12. Szwarcwald CL. Strategies for improving the monitoring of vital events in Brazil. Int J Epidemiol. 2008; 37: 738-44.

13. Rede Interagencial de Informações para a saúde (RIPSA). Indicadores Básicos para saúde no Brasil: conceitos e aplicações / Rede Interagencial de informações para a Saúde Ripsa. 2 ed. Brasília: Organização Pan-Americana da Saúde; 2008. 349 p.

14. Frias PG, Pereira PMH, Andrade CLT, Szwarcwald CL. Sistema de Informações sobre Mortalidade: estudo de caso em municípios com precariedade dos dados. Cad Saúde Pública. 2008; 24: 2257-66.

15. Simões C. A mortalidade infantil na transição da mortalidade no Brasil: um estudo de caso comparativo entre o Nordeste e Sudeste [tese]. Belo Horizonte: Centro de desenvolvimento e planejamento regional da Faculdade de Ciências Econômicas da Universidade de Minas Gerais; 1997.

16. Winkler WE. Methods for evaluating and creating data quality. J Information Systems. 2004; 29: 531-50.

17. Mello Jorge MH, Laurenti R, Gotlieb SLD. Análise da qua- lidade das estatísticas vitais brasileiras: a experiência de implantação do SIM e do SINASC. Ciênc Saúde Coletiva. 2007; 12: 643-54

18. Romero DE. Vantagens e limitações do método demográfico indireto e dos dados da PNAD's 98 para estimativa da mortalidade infantil. In: Encontro da Associação Brasileira de Estudos Populacionais, 13, 2002, Ouro Preto. Anais. Ouro Preto; 2002. Disponível em: http://www.abep.nepo. unicamp.br/docs/anais/pdf/2002/gtsaust3romerotexto.pdf

19. Brass W. Demographic data analysis in less developed countries: 1946-1996. Popul Stud. 1996; 50: 451-67.

20. Brass W. Methods of demographic estimation from incomplete data. Chapel Hill: Laboratories for Population Statistics, University of North Carolina at Chapel Hill; 1975.

21. United Nations. Manual X: Indirect Techniques for Demographic Estimation. Popul Stud. 1983; 81: 74-96.

22. Pujol JM. Nuevas metodologías para evaluar y ajustar datos demográficos. Notas de Población, San José. 1985; 13 (39): 57-73.

23. Coale AJ, Trusell TJ. Estimating the time to which Brass estimates apply. In: Preston SH \& Palloni A. Five tuning Brass type mortality estimates with data on ages of surviving children. Popul Bull United Nations. 1978; 10: 49-54.

24. Palloni A, Heligman L. Re-estimation of structural parameters to obtain estimates of mortality in developing countries. Popul Bull United Nations. 1985; 18: 10-33.

25. United Nations. Step-by-step guide to the estimation of child mortality. Popul Stud. 1990; 89: 83

26. Simões CC. Estimativas da mortalidade infantil por microrregiões e municípios. Brasília: Ministério da Saúde; 1999. $81 \mathrm{p}$

27. Hill AG, David PH. Monitoring changes in child mortality: new methods for use in developing countries. Health Policy Plan. 1988; 3: 214-26.

28. Boerma JT, Sommerfelt AE. Demographic and health surveys (DHS): contributions and limitations. World Health Stat Q. 1993; 46: 222-6.

29. Cavenaghi S. Aspectos metodológicos e comparabilidade com pesquisas anteriores. In: Pesquisa Nacional de Demografia e Saúde da Criança e da Mulher, PNDS 2006; dimensões do processo reprodutivo e da saúde da criança. Ministério da Saúde. Centro Brasileiro de Análise e Planejamento. Brasília: Ministério da Saúde; 2009. p. 1332.

30. Murray CJ, Laakso T, Shibuya K, Hill K, Lopez AD. Can we achieve Millennium Development Goal 4? New analysis of country trends and forecasts of under- 5 mortality to 2015. Lancet. 2007; 370: 1040-54.

31. Korenromp EL, Arnold F, Williams BG, Nahlen BL, Snow RW. Monitoring trends in under-5 mortality rates through national birth history surveys. Int J Epidemiol. 2004; 33: 19

32. Guzman JM. Some problems concerning the selection of the most appropriate mortality model for the indirect estimation of infant mortality. Notas de Población. 1985; 13 (39): $75-103$. 
33. Adetunji JA. Infant mortality levels in Africa: does method of estimation matter? Genus. 1996; 52: 89-106.

34. Burkhalter BR, Miller RI, Silva L, Burleigh E. Variations in estimates of Guatemalan infant mortality, vaccination coverage, and ORS use reported by different sources. Rev Panam Salud Publica. 1995; 29: 1-24.

35. Rede Interagencial de Informações para a Saúde (Ripsa). Indicadores e dados básicos para a saúde (IDB); 2009. [acesso em 31 jan 2011]. http://tabnet.datasus.gov.br/cgi/ idb2009/ matriz.htm

36. Mello Jorge MH, Laurenti R, Gotlieb SLD. O Sistema de Informação sobre Mortalidade-SIM. Concepção, implantação e avaliação. In: A experiência brasileira em sistemas de informação em saúde. Produção e disseminação sobre saúde no Brasil. Brasília: Ministério da Saúde; 2009. v. 1. p. 71-96.

37. Brasil. Ministério da Saúde. Portaria $n^{\circ} 116$ de fevereiro de 2009. Regulamenta a coleta de dados, fluxo e periodicidade de envio das informações sobre óbitos e nascidos vivos para os Sistemas de Informações em Saúde sob gestão da Secretaria de Vigilância em Saúde. Brasília; 2009.
38. Almeida MF, Alencar GP, Novaes HMD, Ortiz LP. Sistema de informação e mortalidade perinatal: conceitos e condições de uso em estudos epidemiológicos. Rev Bras Epidemiol. 2006; 9: 56-68.

39. Lima CRA, Schramm JMA, Coeli CM, Silva MEM. Revisão das dimensões de qualidade dos dados e métodos aplicados na avaliação dos sistemas de informação em saúde. Cad Saúde Pública. 2009; 25: 2095-109.

40. Risi Junior JB. Rede Interagencial de Informações para a Saúde-RIPSA: dez anos de desenvolvimento. In: A experiência brasileira em sistemas de informação em saúde. Produção e disseminação sobre saúde no Brasil. Brasília: Ministério da Saúde; 2009. v. 1. p. 129-42.

Recebido em 20 de agosto de 2010

Versão final apresentada em 10 de novembro de 2011

Aprovado em 14 de novembro de 2011 